\title{
Demanda hídrica e eficiência do uso de água da cana-de-açúcar irrigada no semiárido brasileiro ${ }^{1}$
}

\author{
Thieres G. F. da Silva ${ }^{2}$, Magna S. B. de Moura ${ }^{3}$, Sérgio Zolnier ${ }^{4}$, José M. Soares ${ }^{3}$, \\ Vinícius J. de S. Vieira ${ }^{5}$ \& Walter F. Gomes Júnior ${ }^{5}$
}

\begin{abstract}
RESUMO
Este trabalho objetivou realizar uma análise da eficiência do uso de água em um sistema de produção de cana-de-açúcar irrigada no Submédio do Vale do São Francisco, Brasil. Indicadores que consideram a resposta produtiva da cultura e o desempenho de aplicação de água no sistema de produção foram utilizados e calculados por meio de dados de biomassa seca acumulada, rendimento industrial e a partir dos valores de evapotranspiração de referência (ETo), evapotranspiração da cultura (ETc) e do volume de água que entrou no sistema de produção por precipitação e irrigação $(P+I)$. Verificou-se que a maior eficiência do uso de água pela cultura ocorreu entre o sétimo e o oitavo meses produtivos em virtude do aumento da partição de biomassa para os colmos e do suprimento de radiação e, ainda, da redução da ETc em resposta ao tombamento das plantas. Os indicadores utilizados demonstraram que a demanda de água da cultura foi suprida adequadamente em relação à evapotranspiração máxima. A cana-deaçúcar apresentou uma eficiência de produção de 9,49 kg de colmos, 1,22 kg de açúcar e 875,23 mL de álcool por $\mathrm{m}^{3}$ de ETc e 5,36 kg de colmos, 0,69 kg de açúcar e 494,14 ml de álcool por $\mathrm{m}^{3}$ de $\mathrm{P}+\mathrm{I}$.
\end{abstract}

Palavras-chave: biomassa, evapotranspiração máxima, Submédio do Vale do São Francisco

\section{Water requirement and efficiency of water use of irrigated sugarcane in semi-arid Brazil}

\begin{abstract}
The objective of this work was to accomplish an analysis of the water use efficiency in a production system of irrigated sugarcane located in the Lower-Middle São Francisco river basin, Brazil. Indicators based on the crop yield and the water application performance in the production system were used. These indicators were calculated through accumulated dry biomass, industrial yield data and through the values of reference evapotranspiration, crop evapotranspiration and the water volume that entered in the system by rain and irrigation $(\mathrm{P}+\mathrm{I})$. It was verified that, the highest water use efficiency of crop occured between 7th and 8th month, due to increase in the stalk biomass partition and radiation supply, and due to reduction of ETc in response tumbling of the plants. The used indicators demonstrated that the water demand of crop was adequately in relation to maximum evapotranspiration of crop. The irrigated sugarcane presented a production efficiency of $9.49 \mathrm{~kg}$ of stalks, $1.22 \mathrm{~kg}$ of sugar and 875.23 $\mathrm{mL}$ of alcohol per $\mathrm{m}^{3}$ of ETc and of $5.36 \mathrm{~kg}$ of stalks, $0.69 \mathrm{~kg}$ of sugar and $494.14 \mathrm{~mL}$ of alcohol per $\mathrm{m}^{3}$ of $\mathrm{P}+\mathrm{I}$.
\end{abstract}

Key words: biomass, maximum evapotranspiration, Low-Middle São Francisco river basin

\footnotetext{
Parte da tese de doutorado do primeiro autor

2 UAST/UFRPE, Fazenda Saco, Zona Rural s/n, CEP 56900-000, Serra Talhada, PE. E-mail: thieres@uast.ufrpe.br

Embrapa Semiárido, BR 428, Km 152, Zona Rural, CP 23,CEP 56310-000 Petrolina, PE. Fone: (87) 3862 1711, E-mail: magna@cpatsa.embrapa.br, monteiro@cpatsa.embrapa.br

DEA/UFV, Av. P. H. Holfs, s/n, CEP 36570-000, Viçosa, MG. E-mail: zolnier@ufv.br

5 Empresa Agroindústrias do Vale do São Francisco - Agrovale, BA 210, Km 12, Zona Rural, CEP 48900-000, Juazeiro, BA. Fone: (87) 3612 2900,

E-mail: agrovale@agrovale.com.br
} 


\section{INTRODUÇÃO}

Atualmente, o Brasil é um dos maiores produtores mundiais de cana-de-açúcar e de derivados, como etanol e bagaço, cujos recentes avanços na conversão de cana e melaço em biocombustível têm fortalecido cada vez mais a matriz energética brasileira.

No Submédio do Vale do São Francisco, especificamente no município de Juazeiro, BA, a cana-de-açúcar tem apresentado desempenho relevante em relação a outras áreas de cultivo no País, em decorrência da utilização de tecnologias de irrigação no sistema de produção. Nesta região existem em torno de 18 mil hectares de cana-de-açúcar irrigada com produtividade média igual a $91,2 \mathrm{tha}^{-1}$ superior à do rendimento médio estadual $\left(58,7 \mathrm{tha}^{-1}\right)$ e nacional $\left(76,6 \mathrm{tha}^{-1}\right)$ (IBGE, 2009).

A cana-de-açúcar é uma cultura bem adaptada às condições tropicais e subtropicais com alta disponibilidade de água, nutrientes e radiação (Park et al., 2005; Gilbert et al., 2006; Tejera et al., 2007), produzindo grande quantidade de biomassa. Contudo, o estresse hídrico durante as fases críticas (perfilhamento e início da elongação de colmos) pode resultar em reduções expressivas no rendimento de colmos e de açúcar; entretanto, quando bem aplicado (especificamente durante a fase de maturação), o estresse hídrico aumenta a concentração de sacarose nos colmos (Robertson et al., 1999; Inman-Bamber, 2004). Em decorrência disto, percebe-se que o manejo de água adequado e estratégico durante todo o ciclo da cana-de-açúcar, torna-se um aspecto de grande importância visando melhorar o planejamento e a tomada de decisão da irrigação e, consequentemente, aumentar a eficiência de uso da água do sistema de produção (Inman-Bamber \& Smith, 2005).

Em regiões semiáridas a água é um recurso de fundamental relevância para a produção de cana-de-açúcar (Inman-Bamber \& Mcglinchey, 2003; Watanabe et al., 2004; Wiedenfeld, 2004), ao passo que poucas são as informações sobre a eficiência do uso de água no sistema de produção agrícola, sobretudo no que se refere à produção de sacarose e de álcool (Inman-Bamber et al., 1999; Inmam-Bamber \& Smith, 2005).

Muitos pesquisadores têm proposto indicadores para avaliar a eficiência do uso de água (Bos et al., 1994; Perry, 1996; Molden et al., 1998). Esses indicadores consideram a resposta produtiva da cultura e o desempenho da aplicação de água no sistema de produção em relação à demanda hídrica máxima requerida (Pereira et al., 2002).

O primeiro grupo de indicadores está associado à produtividade da água da cultura $\left(\mathrm{PA}_{\mathrm{C}}\right)$, que expressa a relação entre a quantidade produzida e o volume de água aplicado ou consumido pela cultura (Igbadun et al., 2006; Kahlown et al., 2007). Comumente, este indicador é conhecido como uso eficiente de água; no entanto, a $\mathrm{PA}_{\mathrm{C}}$ considera a fração econômica do rendimento (Detar, 2008; Di Paolo \& Rinaldi, 2008). O segundo grupo de indicadores tem sido utilizado em diferentes áreas agrícolas e considera o suprimento relativo de água (SRA), a evapotranspiração relativa (ER), a fração de redução de água (FRA) e o déficit hídrico da cultura (DHC) (Ahmad et al., 2009; Bastiaanssen et al., 2001; Bos et al., 2005; Karatas et al., 2009).
Com base no exposto objetivou-se, com este trabalho, realizar uma análise da eficiência do uso de água em um sistema de produção de cana-de-açúcar irrigada, visando fornecer informações que auxiliem na melhoria do manejo da água no Semiárido brasileiro.

\section{Material E MÉTODOS}

O experimento foi conduzido em um talhão comercial $\left(9^{\circ} 28^{\prime}\right.$ 07 " S; 40 22' 43”'O; 386,5m) de cana-de-açúcar, variedade RB 92-579, cana soca, localizado no município de Juazeiro, BA, durante os meses de junho/2007 a julho/2008, totalizando 13 meses produtivos. $\mathrm{O}$ ciclo da cultura compreendeu as seguintes fases fenológicas: Fase I (brotação e estabelecimento); Fase II (estabelecimento e perfilhamento); Fase III (crescimento máximo) e Fase IV (maturação), que compreenderam, nesta ordem, as datas 28/05/2007 a 27/06/2007, 28/06/2007 a 14/09/ $2007,15 / 09 / 2007$ a 10/05/2008 e 11/05/2008 a 20/06/2008. O solo da área experimental pertence à classe dos Vertissolos; a área de cultivo foi disposta na direção norte-sul, em fileiras com espaçamento de $1,5 \mathrm{~m}$; a adubação foi realizada com base na análise de solo e com a aplicação de $157,50 \mathrm{~kg} \mathrm{ha}^{-1} \mathrm{e} 500 \mathrm{ml} \mathrm{ha}^{-1}$ do produto comercial uréia e Ajipower ${ }^{\circledR}$, respectivamente; para o controle de ervas espontâneas foi aplicado $1 \mathrm{~L} \mathrm{ha}^{-1}$ dos herbicidas Aminol e Trop e a irrigação foi do tipo superficial, utilizando-se um sistema de condução em tubos janelados. Detalhes do sistema e dos eventos de irrigação podem ser obtidos em Silva (2009).

Calculou-se a evapotranspiração de referência (ETo) com base no método de Penman Monteith padronizado pela FAO, utilizando-se os dados de uma estação meteorológica automática (modelo Davis Vantage Pro2, Hayward, CA, USA) localizada a $3 \mathrm{~km}$ da área experimental. Concomitantemente, a evapotranspiração real da cultura (ETc) foi obtida por meio dos dados de fluxo de calor latente (LE) estimados através do método do balanço de energia com base na razão de Bowen (BERB) (Inman-Bamber \& Mcglinchey, 2003; Teixeira et al., 2008). Os componentes do método BERB foram determinados por meio de medidas realizadas com um saldo radiômetro (NR Lite Net radiometer, Campbell Scientific, Inc. Logan, Utah), dois psicrômetros ventilados com termopares do Tipo $\mathrm{T}$ de Cobre Constantan, ambos instalados em uma torre micrometeorológica de oito metros de altura e três fluxímetros situados em diferentes posições nas linhas de cultivo (HFT3-REBS, Campbell Scientific, Inc. Logan, Utah, USA). A torre foi instalada a $350 \mathrm{~m}$ em relação à bordadura, na direção do vento predominante. $\mathrm{O}$ primeiro psicrômetro foi posicionado a uma altura de 2zom do topo do dossel da cultura (em que: $\mathrm{zom}=$ comprimento de rugosidade da superfície para o momentum), enquanto o segundo estava a 1,5 $\mathrm{m}$ acima do primeiro, conforme sugerido por Inman-Bamber \& McGlinchey (2003). À medida em que a cultura foi crescendo, o saldo radiômetro e os psicrômetros foram deslocados para novos níveis, conforme realizado por Inman-bamber \& Mcglinchey (2003). A aquisição dos dados do método BERB foi realizada por meio de um datalogger (CR10X, Campbell Scientific Inc. Logan, Utah, USA) programado para registrar medidas a cada 60 segundos e 
armazenar médias em intervalos de $15 \mathrm{~min}$. Detalhes sobre a precisão e a calibração dos equipamentos utilizados podem ser obtidos em Silva (2009).

Além da evapotranspiração real da cana-de-açúcar obtida em campo pelo método do BERB, no presente estudo também foi determinada a evapotranspiração máxima da cultura (ETm), que difere teoricamente daquela obtida por meio do produto entre ETo e o valor médio da curva do coeficiente de cultura $\left(\mathrm{Kc}_{\text {med }}\right)$ para determinada fase fenológica, como informado em Doorenbos \& Kassam (1994). Em termos de coeficiente de cultura a curva do gráfico, na Figura 1, representa o $\mathrm{Kc}_{\text {med }}$ e os valores acima e abaixo indicam o $\mathrm{Kc}_{\text {max }}$ e $\mathrm{Kc}_{\text {min }}$, sendo a média aritmética desses dois valores o próprio valor de $\mathrm{Kc}_{\text {med }}$. Assumindo duas situações representadas no gráfico teórico da Figura 1, observa-se que na situação 1, na qual $\mathrm{Kc}_{1}>\mathrm{K}_{\text {med }}$, a ETm é resultado do produto entre o ETo e o $\mathrm{Kc}_{1}$, logo que, nesta situação, o $\mathrm{Kc}_{1}$ se refere ao valor de $\mathrm{Kc}_{\max }$. Por outro lado, na situação 2, em que o $\mathrm{Kc}_{2}$ é menor que o $\mathrm{Kc}_{\text {med }}\left(\mathrm{Kc}_{2}<\right.$ $\mathrm{Kc}_{\text {med }}$, a ETm é resultado do produto entre a ETo e $\mathrm{Kc}_{\text {med }}$, pois nesta situação o $\mathrm{Kc}_{\max }$ será igual ao $\mathrm{Kc}_{\text {med }}$, ou seja, a ETm é aquela que ocorre igual ou acima da curva do $\mathrm{Kc}_{\text {med }}$. Este procedimento foi adotado uma vez que, mesmo num sistema de produção irrigado e a depender das condições meteorológicas, de solo e de manejo, o processo de evapotranspiração das culturas pode ser reduzido, o que induz à existência de valores de ETm maiores que a ETc.

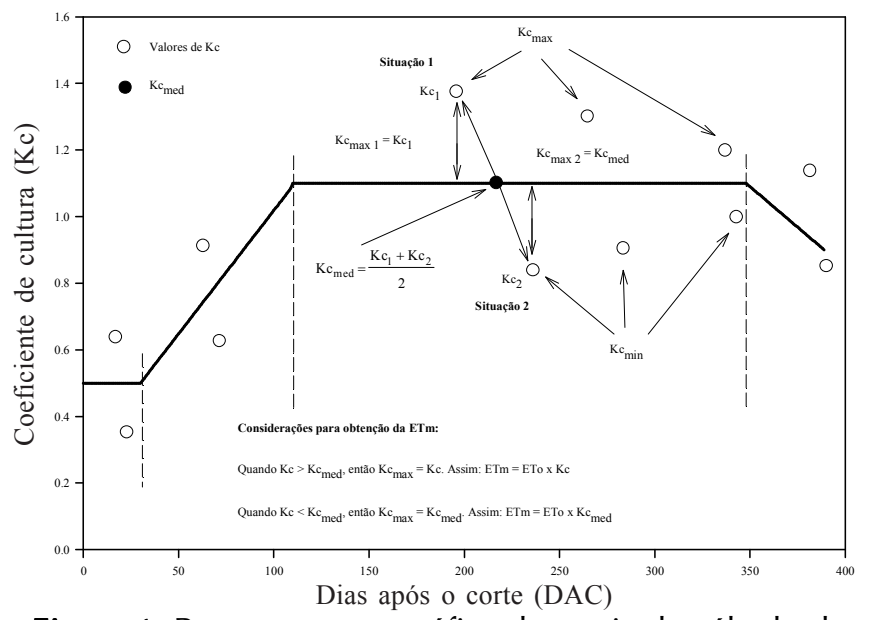

Figura 1. Representação gráfica da teoria de cálculo da evapotranspiração máxima (ETm) a partir dos valores do coeficiente de cultura $(\mathrm{Kc})$

Para determinação da biomassa seca total da parte aérea (BSTPA), dos colmos (BSC) e da palhada (BSPA) da cana-deaçúcar, foi adaptada a metodologia utilizada em vários estudos com cana-de-açúcar (Robertson et al., 1999; Inman-Bamber \& Mcglinchey, 2003; Inman-Bamber, 2004). A partir desta metodologia foram coletados, em três subáreas, perfilhos a nível do solo, em parcelas com quatro a cinco metros lineares, totalizando de 18 a $24 \mathrm{~m}^{2}$ de material, que foram pesados separadamente, obtendo-se o peso total por parcela. Deste volume total, foram amostrados em cada subárea, 15 perfilhos representativos, totalizando 45 perfilhos. Os colmos e a palhada amostrados foram triturados em uma forrageira, de onde foram retiradas subamostras com volumes em torno de $800 \mathrm{~mL}$; em seguida, as subamostras foram colocadas em recipientes de alumínio, pesadas em uma balança de precisão de 0,001 g (Modelo MARK 210A, Bel Engineering, Monza-MI, Itália) para, posteriormente, serem secadas em estufa de ventilação forçada com temperatura entre $65-70^{\circ} \mathrm{C}$. Os valores de biomassa seca total da parte aérea (BSTPA), dos colmos (BSC) e da palhada (BSPA), em $t$ ha $^{-1}$, foram obtidos a partir da extrapolação dos valores dos pesos das subamostras.

A análise da eficiência do uso de água em relação à resposta produtiva da cana-de-açúcar foi realizada por meio da estimativa do uso eficiente de água (UEA) e da produtividade da água da cultura $\left(\mathrm{PA}_{\mathrm{C}}\right)$ calculados para os treze meses produtivos.

Os valores de UEA da cana-de-açúcar foram calculados considerando-se os valores mensais acumulados da biomassa seca da cultura $\left(\mathrm{Y}_{1}\right)$, representados pelo total da parte aérea (BSTPA, $\mathrm{kg} \mathrm{m}^{-2}$ ), da palhada (BSPA, $\mathrm{kg} \mathrm{m}^{-2}$ ) e dos colmos (BSC, $\mathrm{kg} \mathrm{m}^{-2}$ ), bem como pelos valores mensais acumulados de ETc e

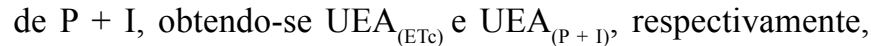
para a BSTPA, PSPA e PSC. A expressão seguinte foi utilizada no cálculo do UEA:

$$
\mathrm{UEA}=\frac{\mathrm{Y}_{1}}{\mathrm{ETc} \mathrm{ou}(\mathrm{P}+\mathrm{I})}
$$

em que:

ETc - valores mensais acumulados da evapotranspiração real da cultura; $\mathrm{P}+\mathrm{I}=$ volume de água que entrou no sistema de produção por precipitação e irrigação, $\mathrm{mm}$.

A PA $_{C}$ é semelhante ao UEA; contudo, considera o rendimento industrializável $\left(\mathrm{Y}_{2}\right)$, em termos de $\mathrm{TCH}$ (toneladas de colmos por hectare), rendimento de açúcar (RBAÇ) e rendimento de álcool (RBAL). Os valores de $\mathrm{PA}_{\mathrm{C}}$ foram quantificados, para o final do ciclo, utilizando-se os valores acumulados de ETc e de $\mathrm{P}+\mathrm{I}$, obtendo $\mathrm{PA}_{\mathrm{C}(\mathrm{ETc})}$ e $\mathrm{PA}_{\mathrm{C}(\mathrm{ETc})}$, respectivamente, para as TCH, RBAÇ e RBAL. A seguinte expressão foi usada:

$$
\mathrm{PA}_{\mathrm{C}}=\frac{\mathrm{Y}_{2}}{\operatorname{ETc~ou}(\mathrm{P}+\mathrm{I})}
$$

O RBAÇ e o RBAL foram calculados a partir dos dados de $\mathrm{TCH}$, percentagem de açúcar bruto e de açúcares redutores livres, conforme métodos laboratoriais e equações citadas por Caldas (1998).

Os valores de UEA e de $\mathrm{PA}_{\mathrm{C}}$ foram determinados tanto por meio dos valores de ETc quanto de P + I, visando comparar a eficiência do uso de água real da cultura, representada por $\mathrm{UEA}_{(\mathrm{P}+\mathrm{I})}$ e $\mathrm{PA}_{\mathrm{C}(\mathrm{P}+\mathrm{I})}$, assumindo-se as perdas de água do sistema de produção da cana-de-açúcar decorrentes das características do sistema de irrigação, solo e técnicas de manejo, com sua eficiência do uso de água sem considerar essas perdas, sendo representada por $\mathrm{UEA}_{(\mathrm{ETc})}$ e $\mathrm{PA}_{\mathrm{C}(\mathrm{ETc})}$.

Para analisar a eficiência do uso de água em relação ao desempenho da aplicação com base na demanda hídrica requerida, calcularam-se os valores mensais dos indicadores: 
suprimento relativo de água (SRA), evapotranspiração relativa (ER), fração de redução de água (FRA) e déficit hídrico da cultura (DHC).

O suprimento relativo de água (SRA) é um indicador utilizado para avaliar se o volume de água aplicado está atendendo à demanda máxima de água da cultura (Perry, 1996). Molden et al. (1998) consideram valor de SRA igual a 2 como sendo o ideal para o sistema de produção e que valores acima deste indicam que um grande volume de água pode estar sendo perdido por drenagem profunda, aumentando o nível do lençol freático. Em contrapartida, o valor de SRA igual a 0,80 é considerado o limite crítico para aumentar o uso eficiente de água sob condições de restrição hídrica (Bos et al., 2005; Teixeira et al., 2008). O SRA foi calculado pela expressão:

$$
\mathrm{SRA}=\frac{(\mathrm{P}+\mathrm{I})}{\mathrm{ETm}}
$$

em que:

ETm - valores mensais acumulados da evapotranspiração máxima da cultura.

A evapotranspiração relativa (ER) quantifica a redução da evapotranspiração real da cultura permitindo que se avalie o desempenho do fornecimento de água ao longo do tempo e se identifiquem as áreas com limitação hídrica (Bandara, 2006). Em áreas irrigadas um valor aceitável de ER está acima de 0,75 (Roerink et al., 1997). A ER foi estimada por:

$$
\mathrm{ER}=\frac{\mathrm{ETc}}{\mathrm{ETm}}
$$

em que:

ETc - valores mensais acumulados da evapotranspiração real da cultura.

O déficit hídrico da cultura (DHC, $\mathrm{mm}$ ) para determinado período é definido como a diferença entre a evapotranspiração real (ETc) e a evapotranspiração máxima (ETm) (Karatas et al., 2009). Para o período de um mês Bastiaanssen et al. (2001) assumem o valor de $30 \mathrm{~mm}$ como aceitável para um sistema de produção. O DHC foi calculado utilizando-se a equação:

$$
\mathrm{DHC}=\mathrm{ETm}-\mathrm{ETc}
$$

A fração de redução de água (FRA) é um indicador que permite demonstrar as variações do consumo de água pela cultura. Para regiões semiáridas o valor crítico de FRA varia entre 0,5 e 0,7 e a faixa aceitável está situada entre 0,6 e 1,1, de modo que valores abaixo de 0,6 indicam que um grande volume de água não está sendo utilizado pela cultura (Bastiaassen et al., 2001; Bos et al., 2005). O valor de FRA foi obtido pela equação:

$$
\mathrm{FRA}=\frac{\mathrm{ETc}}{(\mathrm{P}+\mathrm{I})}
$$

\section{RESULTADOS E DISCUSSÃO}

Em termos de lâminas acumuladas, as entradas (irrigação e precipitação) e saídas (evapotranspiração da cultura - ETc e evapotranspiração máxima - ETm) de água do sistema de produção da cana-de-açúcar, para todas as fases fenológicas, podem ser observadas na Figura 2.

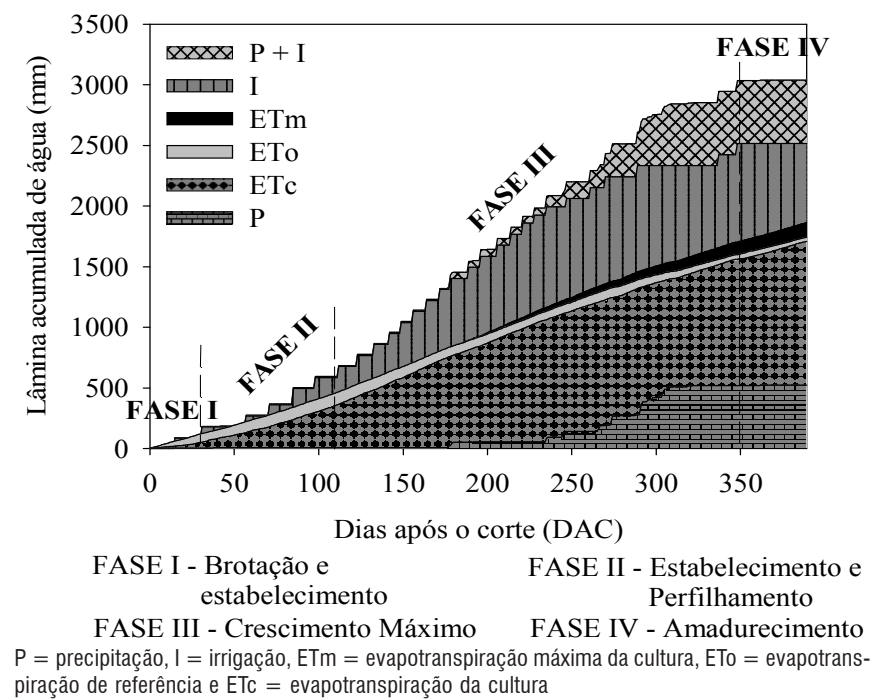

Figura 2. Entradas (irrigação e precipitação) e saídas (evapotranspiração) acumuladas após o corte da cana-deaçúcar irrigada (variedade RB 92579), no ciclo de canasoca, sob as condições climáticas do Submédio do Vale do São Francisco

Verificou-se que a lâmina total de água que entrou no sistema de produção por precipitação e irrigação $(\mathrm{P}+\mathrm{I})$ foi superior a ETc, totalizando 3073 e $1710 \mathrm{~mm}$, respectivamente. Do volume de $\mathrm{P}+\mathrm{I}(3073 \mathrm{~mm}), 2525 \mathrm{~mm}$, ou seja, $82,2 \%$, foram oriundos da irrigação e apenas $523 \mathrm{~mm}$, isto é, 17,8\%, resultantes da precipitação $(\mathrm{P})$, sendo que a maior parte deste valor ocorreu durante a Fase III (crescimento máximo). Constatou-se, ainda, que durante o ciclo a ETc acumulada foi ligeiramente inferior à $\mathrm{ETm}$, que totalizou valores iguais a $1860 \mathrm{~mm}$; finalmente, a demanda atmosférica local, em termos de evapotranspiração de referência (ETo), foi de $1743 \mathrm{~mm}$.

Em termos de produção de biomassa constata-se, na Figura 3, que o máximo incremento de matéria seca total da parte aérea ocorreu entre o sétimo e o oitavo meses produtivos (Fase III), quando foi constatada uma taxa média de $12,47 \pm 0,12 \mathrm{tha}^{-1} \mathrm{mês}^{-1}$, como resposta de um aumento na partição para os colmos, atingindo uma taxa máxima de $10,45 \pm 0,50$ tha $^{-1}$ mês $^{-1}$. Neste período se observou uma elongação mais pronunciada dos colmos, balé devido a maior suprimento de radiação (entre dezembro e janeiro) associada à disponibilidade de água pela irrigação.

Nos períodos que compreenderam a Fase I (brotação e estabelecimento) e a Fase II (estabelecimento e perfilhamento), foram constatados dois padrões de crescimento dos colmos, um nos três primeiros meses produtivos (MP), com a ocorrência de uma taxa média de acúmulo de biomassa pequena $(0,58 \pm 0,15$ t ha ${ }^{-1}$ mês $\mathrm{s}^{-1}$ ) e o segundo se verificando no quarto mês, quando foi evidenciado maior crescimento da cultura $\left(1,55 \mathrm{tha}^{-1} \mathrm{mês}^{-1}\right)$. 


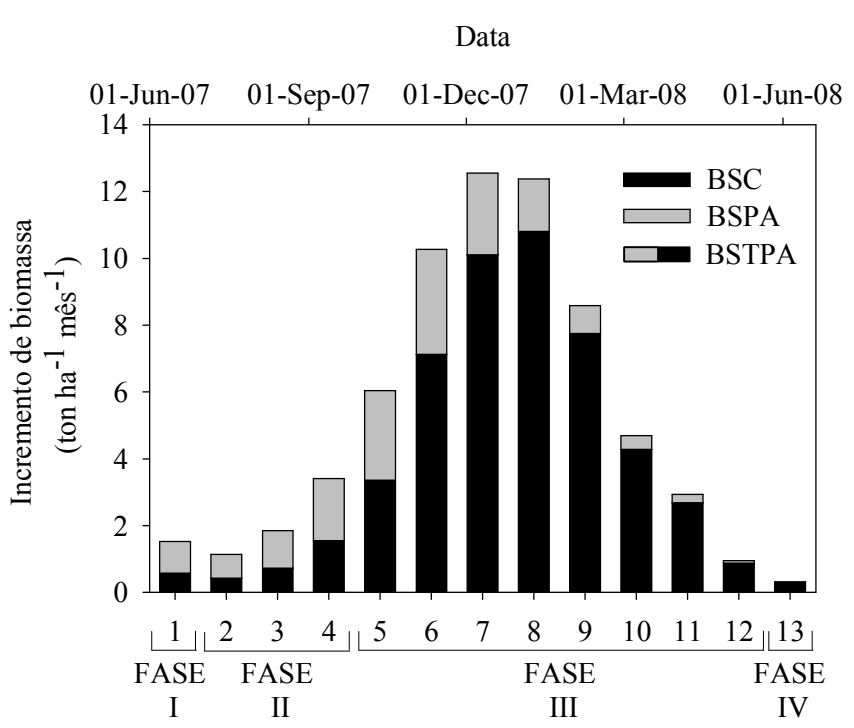

Mês produtivo

Figura 3. Incremento mensal de biomassa seca total da parte aérea (BSTPA) e a partição para os colmos (BSC) e palhada (BSP, peso total menos o peso dos colmos) da cultura da cana-de-açúcar cultivada na região semiárida da Bacia do Rio São Francisco

Em decorrência da maior partição para os colmos, a biomassa destinada à palhada começou a reduzir a partir do sétimo mês (MP7); até antes deste mês produtivo a biomassa destinada à palhada havia aumentado de 0,95 a $3,14 \mathrm{t} \mathrm{ha}^{-1}$ mês $^{-1}$, enquanto a partir do MP7 a taxa de crescimento reduziu de 2,45 $\mathrm{t} \mathrm{ha}^{-1} \mathrm{mês}^{-1}$ para $0,03 \mathrm{t} \mathrm{ha}^{-1} \mathrm{mês}^{-1}$, no último produtivo (13 MP). Para a biomassa total da parte aérea e dos colmos a taxa diminuiu para 0,33 e 0,30 t ha $^{-1}$ mês $^{-1}$ no MP13, respectivamente. Em média, durante o ciclo o crescimento médio foi de 5,13, 3,89 e 1,24 tha ${ }^{-1}$ mês $^{-1}$ para a parte área, colmos e palhada, respectivamente.

Em termos de partição, na Fase I a biomassa de colmos e a palhada corresponderam a 37 e $63 \%$ do peso total nessa ordem, variando até $40 \%$ e $60 \%$ na Fase II, enquanto na Fase III a biomassa destinada aos colmos respondeu a até $76 \%$, apresentando pouco incremento até o final do ciclo (Fase IV).

$\mathrm{O}$ uso eficiente da água (UEA) mensal da cana-de-açúcar em relação à ETc e $(\mathrm{P}+\mathrm{I})$, pode ser observada nas Figuras $4 \mathrm{~A} \mathrm{e}$ $4 \mathrm{~B}$, respectivamente.

Com base nessas figuras, constatou-se que os meses produtivos 7 (MP7) e 8 (MP8) (compreendidos na Fase III) apresentaram os maiores valores de $\mathrm{UEA}_{\mathrm{BSTP}}$, sendo de 7,88 \pm $0,10 \mathrm{~kg} \mathrm{~m}^{-3}$ em relação à ETc e de 4,01 $\pm 0,70 \mathrm{~kg} \mathrm{~m}^{-3}$ quando relacionados com $(\mathrm{P}+\mathrm{I})$. Verificou-se, também, que neste período ocorreram os maiores valores de $\mathrm{UEA}_{\mathrm{BSC}}$, que foram de $6,60 \pm 0,29 \mathrm{~kg} \mathrm{~m}^{-3}$ em relação à ETc e de $3,35 \pm 0,39 \mathrm{~kg} \mathrm{~m}^{-3}$ quando relacionados com $(\mathrm{P}+\mathrm{I})$; no período subsequente e dentro desta mesma fase, os valores de $\mathrm{UEA}_{\mathrm{BSC}}$ reduziram de $0,81 \mathrm{~kg} \mathrm{~m}^{-3}$ para $0,48 \mathrm{~kg} \mathrm{~m}^{-3}$ (Figura 4); na Fase IV (MP13), o valor de $\mathrm{UEA}_{\mathrm{BSC}}$ foi de $9,71 \mathrm{~kg} \mathrm{~m}^{-3}$, considerando-se (P+I) (Figura 4B), em decorrência do longo período de suspensão da irrigação e do pequeno acúmulo de biomassa; por outro lado, quando se considerou a ETc constatou-se o menor valor $\left(0,26 \mathrm{~kg} \mathrm{~m}^{-3}\right)$ dentre os meses produtivos; para o ciclo, como um todo, verificou-se que os valores de $\mathrm{UEA}_{\mathrm{BSC}}$ foram de 2,95 e de 1,67 $\mathrm{kg} \mathrm{m}^{-3}$, em relação à ETc e a $(\mathrm{P}+\mathrm{I})$, respectivamente (Tabela 1).

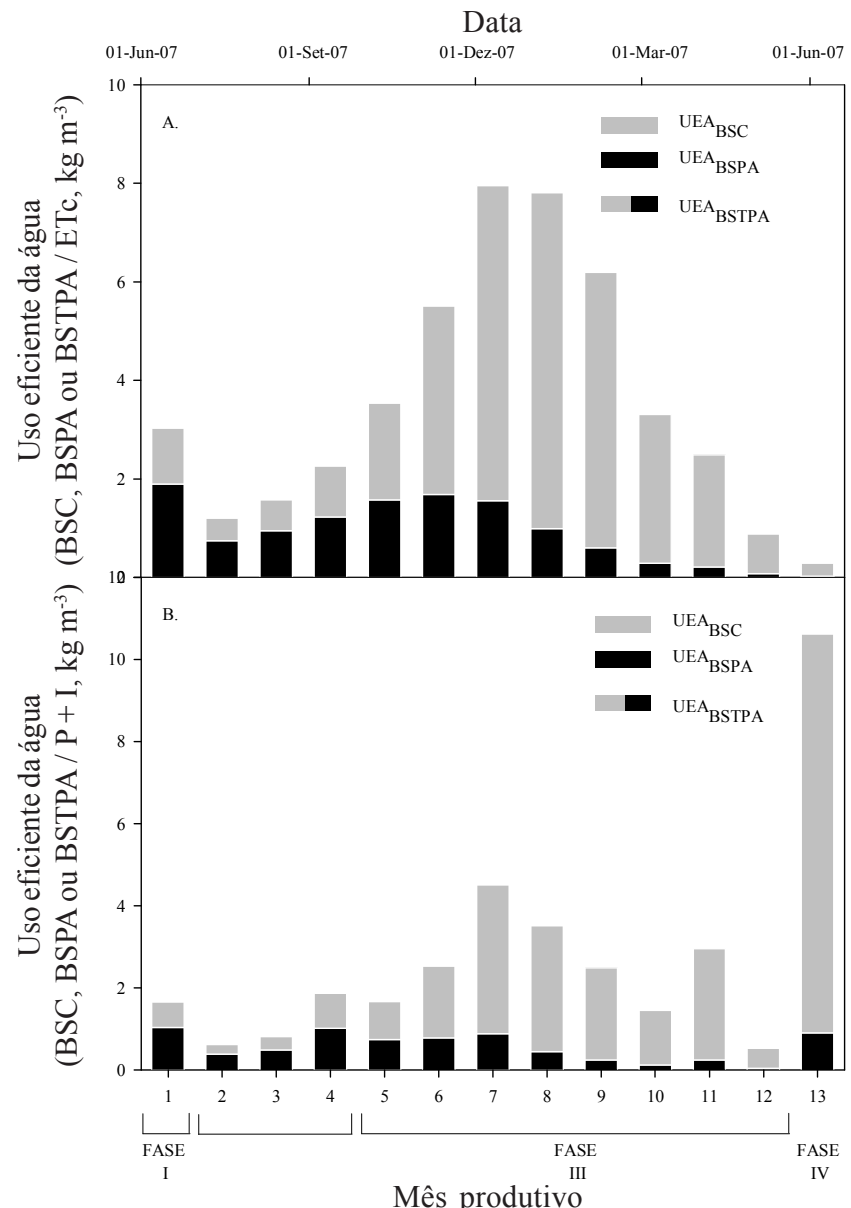

Figura 4. Uso eficiente de água (UEA) para os meses produtivos da cana-de-açúcar irrigada, no ciclo de canasoca, sob as condições climáticas do Submédio do Vale do São Francisco, em relação à biomassa seca dos colmos (BSC), palhada (BSPA) e biomassa seca total da parte aérea (BSTPA), considerando-se a evapotranspiração da cultura (ETC) (A) e o volume de água que entrou no sistema por precipitação e irrigação $(P+I)(B)$

Tabela 1. Indicadores da eficiência do uso de água para a cana-de-açúcar irrigada (variedade RB 92579), no ciclo de cana-soca, sob as condições climáticas do Submédio do Vale do São Francisco

\begin{tabular}{|c|c|}
\hline UEA $_{(\mathrm{ETC})}$, em base seca da parte aérea $\left(\mathrm{kg} \mathrm{m}^{-3}\right)$ & 3,93 \\
\hline $\mathrm{UEA}_{(\mathrm{P}+\mathrm{l})}$, em base seca da parte aérea $\left(\mathrm{kg} \mathrm{m}^{-3}\right)$ & 2,22 \\
\hline UEA $_{(\mathrm{ETC})}$, em base seca de colmos $\left(\mathrm{kg} \mathrm{m}^{-3}\right)$ & 2,95 \\
\hline$U_{E A}(P+I)$, em base seca de colmos $\left(\mathrm{kg} \mathrm{m}^{-3}\right)$ & 1,67 \\
\hline $\mathrm{UEA}_{(\mathrm{ETC})}$, em base seca de palhada $\left(\mathrm{kg} \mathrm{m}^{-3}\right)$ & 0,94 \\
\hline$U_{E A_{(P+l)}}$, em base seca de palhada $\left(\mathrm{kg} \mathrm{m}^{-3}\right)$ & 0,53 \\
\hline $\mathrm{PA}_{\mathrm{C}(\mathrm{ETC})}$, em base industrial de colmos $\left(\mathrm{kg} \mathrm{m}^{-3}\right)$ & 9,49 \\
\hline $\mathrm{PA}_{\mathrm{C}(\mathrm{P}+\mathrm{l})}$, em base industrial de colmos $\left(\mathrm{kg} \mathrm{m}^{-3}\right)$ & 5,36 \\
\hline $\mathrm{PA}_{\mathrm{C(ETC)}}$, em base de açúcar $\left(\mathrm{kg} \mathrm{m}^{-3}\right)$ & 1,22 \\
\hline $\mathrm{PA}_{\mathrm{C}(\mathrm{P}+\mathrm{l})}$, em base de açúcar $\left(\mathrm{kg} \mathrm{m}^{-3}\right)$ & 0,69 \\
\hline $\mathrm{PA}_{\mathrm{C}(\mathrm{ET} \mathrm{C})}$, em base de álcool $\left(\mathrm{ml} \mathrm{m}^{-3}\right)$ & 875,23 \\
\hline $\mathrm{PA}_{\mathrm{C}(\mathrm{P}+\mathrm{l})}$, em base de álcool $\left(\mathrm{ml} \mathrm{m}^{-3}\right)$ & 494,14 \\
\hline SRA (adimensional) & 1,63 \\
\hline $\mathrm{ER}(\mathrm{mm})$ & 0,92 \\
\hline FRA (adimensional) & 0,56 \\
\hline $\mathrm{DHC}(\mathrm{mm})$ & 145,30 \\
\hline
\end{tabular}


Em base de palhada notou-se, para a Fase III, que os valores máximos de $\mathrm{UEA}_{\mathrm{BSPA}}$ ocorreram entre os meses produtivos 4 (MP4) e 7 (MP7), tendo sido de 1,51 $\pm 0,20$ e de $0,85 \pm 0,12 \mathrm{~kg} \mathrm{~m}^{-3}$, correspondentes à ETc e a $(\mathrm{P}+\mathrm{I})$, respectivamente (Figuras $4 \mathrm{~A}$ e 4B); em seguida, observou-se diminuição dos valores de $\mathrm{UEA}_{\mathrm{BSPA}}$, como resposta da maior fração de biomassa destinada aos colmos (Figura 3); a partir deste período notou-se que seus valores reduziram até o MP12, passando de 0,99 para 0,07 $\mathrm{kg} \mathrm{m}^{-3}$, quando relacionados com a ETc e de 0,90 para $0,04 \mathrm{~kg} \mathrm{~m}^{-3}$, quando considerados $(\mathrm{P}+\mathrm{I})$. Verificou-se, também, que a $\mathrm{UEA}_{\mathrm{BSPA}}$ apresentou um comportamento de modo semelhante à $\mathrm{UEA}_{\mathrm{BSC}}$, logo que seus valores mais elevados alcançaram $0,90 \mathrm{~kg} \mathrm{~m}^{-3}$; ao final do ciclo o valor de $\mathrm{UEA}_{\mathrm{BSPA}}$ foi de $0,94 \mathrm{~kg} \mathrm{~m}^{-3}$ em relação à ETc e de $0,53 \mathrm{~kg} \mathrm{~m}^{-3}$ quando relacionado com $(\mathrm{P}+\mathrm{I})$ (Tabela 1).

Por outro lado, quando se relacionou o rendimento de colmos em base industrial (TCH), RBAÇ e RBAL com os valores de ETc e de $(\mathrm{P}+\mathrm{I})$, a eficiência do uso de água foi analisada em termos de produtividade da água da cultura $\left(\mathrm{PA}_{\mathrm{C}}\right)$. Observou-se que o valor de $\mathrm{PA}_{\mathrm{C}}$ foi igual a $9,49 \mathrm{~kg} \mathrm{~m}^{-3}$, utilizando-se a ETc, enquanto foi de $5,30 \mathrm{~kg} \mathrm{~m}^{-3}$, quando se considerou (P+I) (Tabela 1).

Inman-Bamber \& Smith (2005) mencionam que a $\mathrm{PA}_{C}$ da cana-de-açúcar pode atingir valores elevados variando entre 4,8 a 12,1 $\mathrm{kg} \mathrm{m}^{-3}$ para o Sudeste da África, Havaí e Austrália, e de 5,3 a 15,4 $\mathrm{kg} \mathrm{m}^{-3}$, a depender da faixa de acúmulo de biomassa da cultura e do déficit de pressão de vapor da região. Singh et al. (2007), avaliando o planejamento da irrigação para cana-deaçúcar (variedade CoPant 84212), com base na relação entre as lâminas de irrigação e da evapotranspiração acumulada do tanque classe $\mathrm{A}$, obtiveram valores de $\mathrm{PA}_{\mathrm{C}}$ variando entre 5,8 e $7,6 \mathrm{~kg} \mathrm{~m}^{-3}$. Doorembos \& Kassam (1994) citam que a cana-deaçúcar sob condições irrigadas nos trópicos e subtrópicos secos, em solos com $80 \%$ de água disponível, pode apresentar entre 5 e $8 \mathrm{~kg} \mathrm{~m}^{-3}$, em termos de colmos e de 0,6 a $1 \mathrm{~kg} \mathrm{~m}^{-3}$, e de sacarose.

Em termos de rendimento de açúcar e de álcool, a cana-deaçúcar irrigada apresentou uma $\mathrm{PA}_{\mathrm{C}}$ de $1,22 \mathrm{~kg} \mathrm{~m}^{-3}$ e 875,23 $\mathrm{ml} \mathrm{m}^{-3}$ quando foram considerados os valores de ETc (Tabela 1). Quando se considerou a lâmina total aplicada $(\mathrm{P}+\mathrm{I})$, seus valores foram de $0,69 \mathrm{~kg} \mathrm{~m}^{-3}$ e $494,14 \mathrm{ml} \mathrm{m}^{-3}$, respectivamente (Tabela 1). Farias et al. (2008) relatam, para a região norte paraibana dos tabuleiros costeiros, que as eficiências do uso de água da cana-de-açúcar (variedade SP 791011), quanto às produções de colmo e de açúcar, foram da ordem de 7,12 e 0,67 $\mathrm{kg} \mathrm{m}^{-3}$, respectivamente, cujos resultados indicam que a canade-açúcar, variedade RB 92597 apresenta, quando bem irrigada sob as condições semiáridas do Nordeste, alta eficiência de produção de açúcar e álcool em comparação com outras regiões e variedades.

Os indicadores de eficiência do uso de água (SRA, ER, DHC e FRA), em relação ao desempenho de aplicação com base na demanda máxima requerida pela cultura, podem ser observados nas Figuras 5 e 6 . O suprimento relativo de água (SRA) variou entre 0,83 e 2,19 ao longo dos meses produtivos (MP) das Fases I, II, III e IV (Figura 5A). No MP1 (junho/2007), verificouse que o valor de SRA foi de 1,15, como resultado do baixo regime de chuvas nesse período e do grande intervalo entre os dois eventos consecutivos de irrigação.

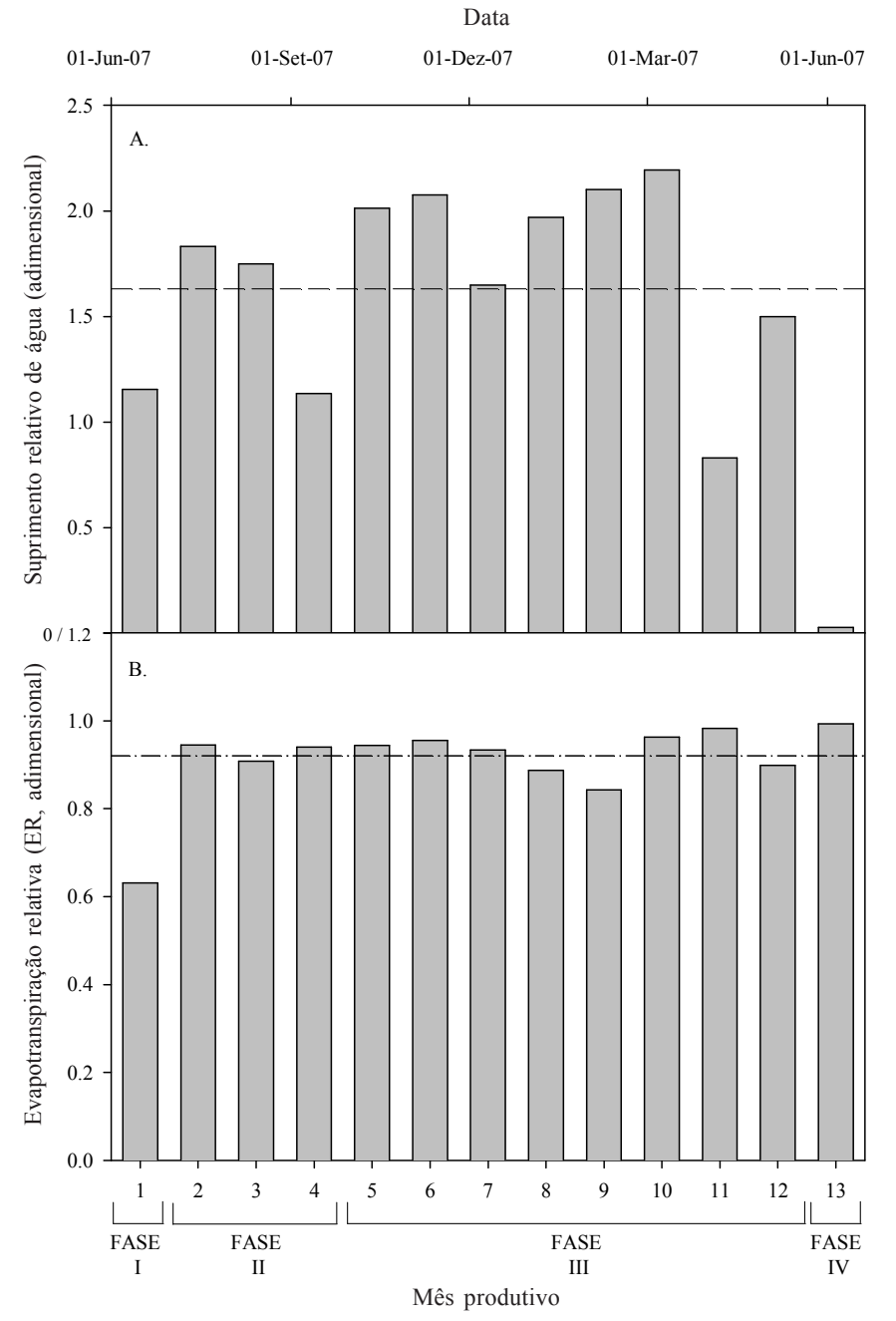

Obs.: A linha tracejada indica o valor médio do ciclo

Figura 5. Suprimento relativo de água (SRA) e evapotranspiração relativa (ER) para os meses produtivos da cana-de-açúcar irrigada (variedade RB 92579), no ciclo de cana-soca, sob as condições climáticas do Submédio do Vale do São Francisco

Nos MP2 e MP3, os valores de SRA são mais altos do que o MP1, sendo iguais a 1,83 e 1,75, mas decresceram para 1,14 no MP4 (setembro/2007) (Figura 5A). Este comportamento se deu em decorrência do aumento da demanda máxima da cultura, como reposta da elevação da demanda atmosférica local; entretanto, na Fase III, os valores de MP5 a MP10 foram, em média, iguais a 2,0 $\pm 0,19$. Contudo, o MP10 ocorreu dentro do período mais chuvoso desta região que, combinado com a redução da disponibilidade de radiação solar e, por consequente, diminuição da demanda atmosférica, apresentou diminuição da demanda máxima pela cultura.

Assim, o volume de água adicionado ao sistema por precipitação e irrigação, resultou em elevados valores de SRA $(2,19)$ (Figura 5A). Segundo Molden et al. (1998), valores de SRA em torno de 2 são considerados ideais para o sistema de produção e valores muito acima indicam que um grande volume de água pode estar sendo perdido por drenagem profunda, aumentando o nível do lençol freático ou sendo perdido escoamento; já o MP11 ocorreu no período final da estação 


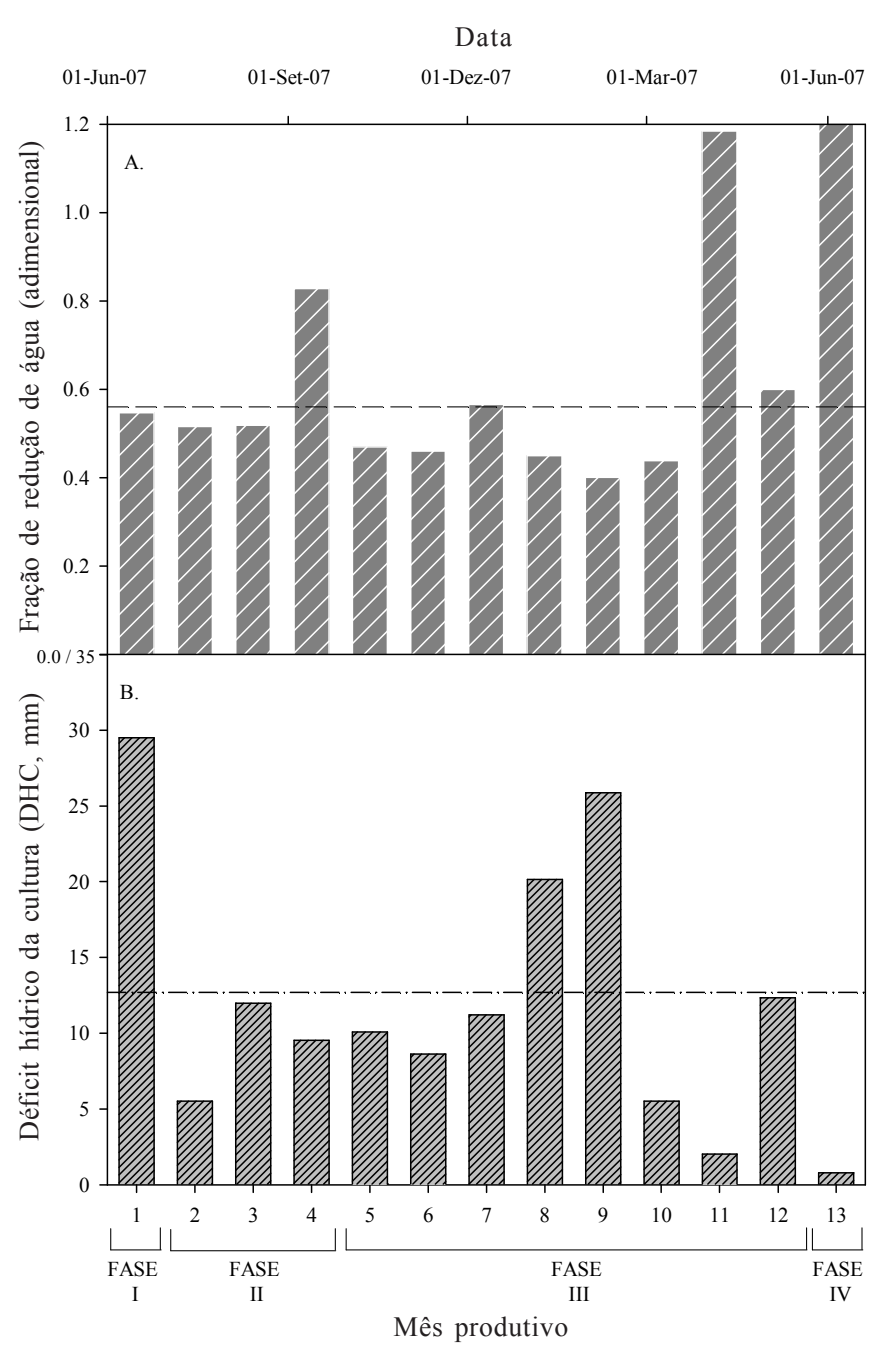

Obs.: A linha tracejada indica o valor médio do ciclo

Figura 6. Fração de redução de água (FRA) - (A) e déficit hídrico da cultura (DHC) - (B) para os meses produtivos da cultura da cana-de-açúcar irrigada (variedade RB 92579), no ciclo de cana-soca, sob as condições climáticas do Submédio do Vale do São Francisco

chuvosa, quando então o número de eventos de irrigação era reduzido (dados não mostrados). Apesar disto, a cana-deaçúcar apresentou uma UEA $_{\text {BSTPA }}$ de $2,49 \mathrm{~kg} \mathrm{~m}^{-3}$ (Figura 4A), indicando que um SRA igual a 0,83 foi ainda bastante para aumentar a eficiência do uso de água pela cultura.

Resultados similares foram observados por Teixeira et al. (2008), para a cultura da mangueira, os quais obtiveram um SRA igual a 0,80 , considerado limite crítico para promover o aumento da eficiência do uso de água pela cultura. No MP12 (maio/2008), ocorreram apenas dois eventos de irrigação que foram realizados no começo do mês e permitiram a reposição de água ao solo, mas, na sequência, a irrigação foi suspensa, resultando no valor de SRA igual 1,50; além disso, verificou-se redução da demanda máxima da cultura (ETm). O valor de SRA mais baixo $(0,03)$ foi obtido no último mês produtivo (MP13), em decorrência do elevado déficit de água no solo, causado pela suspensão da irrigação.

O valor médio obtido para o ciclo da cana-de-açúcar foi de 1,63 (Tabela 1) situado, portanto, na faixa de valores de SRA informados por Karatas et al. (2009), para os distritos de Salihli RB $(1,66)$ e Salihli LB $(1,24)$ localizados na Turquia. Dentre os distritos avaliados pelos autores esses apresentaram os melhores desempenhos quanto ao fornecimento de água para as culturas.

Quando se analisou a evapotranspiração relativa (ER) (Figura 5B) constatou-se, entre os meses produtivos, que os menores valores foram o MP1 (junho/2007) com 0,63, MP8 (janeiro/2008) com 0,89 e MP9 (fevereiro/2008) com 0,84. Roerink et al. (1997) citam que os valores aceitáveis de ER para áreas irrigadas devem ser superiores a 0,75 .

Com base neste valor de referência, apenas os MP8 e MP9 se situaram acima de 0,75 ; contudo, o valor de 0,63 pode ser considerado aceitável, visto que o $\mathrm{UEA}_{\mathrm{BSTPA}}$ se situou em torno de $3,03 \mathrm{~kg} \mathrm{~m}^{-3}$. Constatou-se também que, apesar da restrição do conteúdo de água condicionado pela suspensão da irrigação no período de maturação da cultura (MP13), a ETc alcançou valores muito próximos ao máximo esperado para esta fase, resultando em um valor de ER igual a 0,97 (Figura 5B); este resultado está associado à demanda máxima da cultura da canade-açúcar, que foi relativamente baixa neste período, devido à elevada capacidade de retenção de água dos vertissolos; assim e mesmo sob condições de suspensão de água, o volume de água armazenado no solo atendeu à demanda evapotranspirométrica desta cultura na sua fase final de maturação.

Os MP8 e MP9 compreenderam meses em que a demanda máxima da cultura foi considerada alta, apesar da diminuição dos valores de ER. Este resultado é decorrente da redução de ETc em virtude do tombamento sofrido pela cultura. Ao longo do ciclo o valor médio de ER foi de 0,92 (Tabela 1). Bastiaanssen et al. (2001) observaram valores de 0,76 para fruteiras cultivadas no Submédio do Vale do São Francisco, enquanto Karatas et al. (2009) encontraram valores médios em torno de 0,70 para dez distritos irrigados na Turquia.

A fração de redução de água (FRA) (Figura 6A) revelou um valor médio para o ciclo da cana-de-açúcar igual a 0,56 (Tabela 1 ), indicando que apenas $56 \%$ do volume total de água $(\mathrm{P}+\mathrm{I})$ que entrou no sistema de produção, foram utilizados pela cultura. Este valor de FRA está dentro da faixa limite de 0,5 a 0,7 para regiões de clima semiárido (Bastiaanssen et al., 2001). Isto significa que $44 \%$ da lâmina de água aplicada deixaram de ser efetivamente utilizados, tornando-se importante a redistribuição da aplicação de água para outros períodos do ciclo da cultura (Bos et al., 2005). Analisando-se a FRA para cada mês produtivo, observaram que dez dos 13 meses produtivos apresentaram excesso de água, uma vez que os valores de FRA foram inferiores a 0,6 (valor médio limite); entretanto, na Fase IV os valores de FRA se mostraram bastante elevados (Figura 6A), logo que a entrada de água no sistema por $\mathrm{P}+\mathrm{I}$ foi de $3 \mathrm{~mm}$, proveniente apenas de dois eventos de precipitação.

Como o indicador DHC está relacionado com o SRA (Figura 6B), é possível conhecer a magnitude da deficiência hídrica sofrida pela cultura ao longo dos meses produtivos, ou seja, quanto a mesma deixou de evapotranspirar em relação à sua demanda máxima (Bastiaanssen et al., 2001). Apesar dos altos valores de SRA obtidos nos MP8 e MP9, decorrentes do alto volume de água que entrou no sistema, constatou-se deficiência 
de água como resposta especialmente do tombamento da cultura, que condicionou uma pequena redução do seu IAF (dados não mostrados) e, consequentemente, na evapotranspiração da cana-de-açúcar em relação à sua demanda máxima; além do mais, a redução da ETc pode estar relacionada a fatores intrínsecos do solo e da cultura, como retenção de água no solo e controle estomático, ou das condições meteorológicas vigentes, restringindo a absorção de água pelas plantas.

Esta deficiência foi pequena, atingindo valores de 20,2 $\mathrm{mm}$ mês ${ }^{-1}$ para o MP8 e de 25,9 mm mês ${ }^{-1}$ para MP9, sendo em média igual a $23,0 \pm 4,0 \mathrm{~mm}^{\text {mês }}{ }^{-1}$, e favoreceu o aumento da eficiência do uso de água da cana-de-açúcar entre o MP7 e MP8 no MP1; enfim, verificaram-se valores de DHC atingindo $29,5 \mathrm{~mm} \mathrm{mês}^{-1}$, os quais se mostraram inferiores ao informado por Bastiaanssen et al. (2001) que assumem, como valor crítico de DHC, $30 \mathrm{~mm}^{\text {mês }}{ }^{-1}$.

O DHC acumulado ao longo do ciclo foi de apenas 145,3 $\mathrm{mm}$, correspondendo a $8,5 \%$ da demanda máxima requerida pela cultura (Tabela 1). No MP1 (Fase I), em que a restrição hídrica foi de $29,5 \mathrm{~mm}^{\text {mês }}{ }^{-1}$, a equivalência foi de $58,4 \%$ em relação à sua demanda máxima acumulada para esta fase; no entanto, alcançou 89,15 mm mês ${ }^{-1}(7,4 \%)$, na Fase III e apenas $11,0 \mathrm{~mm}$ mês ${ }^{-1}$ ou $7,4 \%$ da sua demanda máxima acumulada na Fase IV.

\section{CONCLUSÃo}

1. A maior produção de palhada da cana-de-açúcar irrigada ocorreu até o sexto mês produtivo quando, a partir daí, se constatou uma eficiência maior do uso de água pela cultura, ocorrendo entre os sétimo e oitavo meses, resultado do aumento da partição de biomassa para os colmos e do suprimento de radiação e devido à redução da ETc em decorrência do tombamento da cultura.

2. A eficiência do uso de água da cana-de-açúcar a partir do desempenho da aplicação de água demonstrou que a cultura apresentou déficit hídrico, em alguns meses produtivos, decorrente do tombamento da cultura.

3. A cana-de-açúcar irrigada apresentou padrões elevados de eficiência de produção, tanto quando se considerou o volume de água evapotranspirada pela cultura $(9,49 \mathrm{~kg}$ de colmos, 1,22 kg de açúcar e 875,23 ml de álcool por metro cúbico) quanto à lâmina de água que entrou na área de cultivo por precipitação e irrigação $(5,36 \mathrm{~kg}$ de colmos, $0,69 \mathrm{~kg}$ de açúcar e 494,14 ml de álcool por metro cúbico).

\section{LiTERATURA CITADA}

Ahmad, M. D.; Turral, H.; Nazeer, A. Diagnosing irrigation performance and water productivity through satellite remote sensing and secondary data in a large irrigation system of Pakistan. Agricultural Water Management, v.96, p.551-564, 2009.

Bandara, K. M. P. S. Assessing irrigation performance by using remote sensing. Wageningen: Wageningen University. 2006. 156p. Doctoral thesis
Bastiaanssen, W. G. M.; Brito, R. A. L.; Bos, M. G.; Souza, R. A.; Cavalcanti, E. B.; Bakker, M. M. Low cost satellite data for monthly irrigation performance monitoring: Benchmarks from Nilo Coelho, Brazil. Irrigation Drainage System, v.15, p.5379, 2001.

Bos, M. G.; Burton, M. A.; Molden, D. J. Performance indicators for irrigation and drainage, In: Irrigation and drainage performance assessment, practical guidelines. Cambridge: CABI Publishing, 2005. p.26-61.

Bos, M. G.; Murray-Rust, D. H.; Merrey, D. J.; Johnson, H. G.; Snellen,W. B. Methodologies for assessing performance of irrigation and drainage management. Irrigation and Drainage Systems, v.5, 1994. p.231-261.

Caldas, C. Manual de análises selecionadas para indústrias sucroalcooleiras. Maceió: Sindicato da Indústria e do Álcool do Estado de Alagoas, 1998. 438p.

Detar, W. R. Yield and growth characteristics for cotton under various irrigation regimes on sandy soil. Agricultural Water Management, v.95, p.69-76, 2008.

Di Paolo, E. D.; Rinaldi, M. Yield response of corn to irrigation and nitrogen fertilization in a Mediterranean environment. Field Crops Research, v.105, p.202-210, 2008.

Doorenbos, J.; Kassam, A. H. Efeito da água no rendimento das culturas. Campina Grande: UFPB, 1994. 306p. Estudos FAO. Irrigação e Drenagem, 33

Farias, C. H. A.; Fernandes, P. D.; Dantas Neto, J. Y.; Gheyi, H. R. Eficiência no uso da água na cana-de-açúcar sob diferentes lâminas de irrigação e níveis de zinco no litoral paraibano. Engenharia Agrícola, v.28, p.494-506, 2008.

Gilbert, R. A.; Shine Jr., J. M.; Miller, J. D.; Rainbolt, C. R. The effect genotype, environmental and time of harvest on sugarcane yields in Florida, USA. Field Crops Research, v.95, p.156-170, 2006.

IBGE - Instituto Brasileiro de Geografia e Estatística. Produção agrícola 2007: Culturas perenes. http://www.ibge.gov.br. 18 Abr. 2009.

Igbadun, H. E.; Mahoo, H. F.; Tarimo, A. K. P. R.; Baanda, A. S. Crop water productivity of an irrigated maize crop in Mkoji sub-catchment of the Great Ruaha River Basin, Tanzania. Agricultural Water Management, v.85, p.141-150, 2006.

Inman-Bamber, N. G. Sugarcane water stress criteria for irrigation and drying off. Field Crops Research, v.89, p.107-122, 2004.

Inman-Bamber, N. G.; Mcglinchey, M. G. Crop coefficients and water-use estimates for sugarcane based on long-term Bowen ratio energy balance measurements. Field Crops Research, v.83, p.125-138, 2003.

Inman-Bamber, N. G.; Robertson, M. J.; Muchow, R. C.; Wood, A. W. Efficient use of water resources in sugar production: a physiological basis for crop response to water supply. Aitkenvale: CSIRO Tropical Agriculture, 1999.38p. Relatório final

Inman-Bamber, N. G.; Smith, D. M. Water relations in sugarcane and response to water deficits, Field Crops Research, v.92, p.185-202, 2005.

Kahlown, M. A.; Raoof, A.; Zubair, M.; Kemper, W. D. Water use efficiency and economic feasibility of growing rice and wheat with sprinkler irrigation in the Indus Basin of Pakistan. Agricultural Water Management, v.87, p.292-298, 2007. 
Karatas, B. S.; Akkuzu, E.; Unal, H. B.; Asik, S.; Avci, M. Using satellite remote sensing to assess irrigation performance in Water User Associations in the Lower Gediz Basin, Turkey. Agricultural Water Management, v.96, p.982-990, 2009.

Molden, D. J.; Sakthivadivel, R.; Perry, C. J.; Fraiture, C.; Kloezen, W.H. Indicators for Comparing Performance of Irrigated Agricultural Systems. Sri Lanka: International Water Management Institute (IWMI), 1998. 34p.

Park, S. E.; Robertson, M.; Inman-Bamber, N. G. Decline in the growth of a sugarcane crop with age under high input conditions. Field Crops Research, v. 92, p.305-320, 2005.

Pereira, L. S.; Oewis, T.; Zairi, A. Irrigation managament under water scarcity. Agricultural Water Management, v.57, p.175206, 2002.

Perry, C. J. Quantification and measurement of a minimum set of indicators of the performance of irrigation systems. Sri Lanka: International Water Management Institute (IWMI), 1996.

Robertson, M. J.; Inman-Bamber, N. G.; Muchow, R. C.; Wood, A. W. Physiological analysis of early season deficit and sugarcane productivity. Field Crops Research, v.64, p.211227, 1999.

Roerink, G. J.; Bastiaanssen, W. G. M.; Chambouleyron, J.; Menenti, M. Relating crop water consumption to irrigation water supply by remote sensing. Water Resources Management, v.11, p.445-465, 1997.
Silva, T. G. F. da. Análise de crescimento, interação biosferaatmosfera e eficiência do uso de água da cana-de-açúcar irrigada no Submédio do Vale do São Francisco. Viçosa: UFV, 2009. 194p. Tese Doutorado

Singh, P. N.; Shukla, S. K.; Bhatnagar, V. K. Optimizing soil moisture regime to increase water use efficiency of sugarcane (Saccharum spp. hybrid complex) in subtropical India. Agricultural Water Management, v.90, p.95-100, 2007.

Teixeira, A. H. de C.; Bastiaanssen, W. G. M.; Moura, M.S.B.; Soares, J. M.; Ahmad, M. D.; Bos, M. G. Energy and water balance measurements for water productivity analysis in irrigated mango trees, Northeast Brazil. Agricultural and Forest Meteorology, v.14, p.1524-1537, 2008.

Tejera, N. A.; Rodés, R.; Ortega, E.; Campos, R.; Lluch, C. Comparative analysis of physiological characteristics and yield components in sugarcane cultivars. Field Crops Research, v.102, p.64-72, 2007.

Watanabe, K.; Yamamoto, T.; Takashi Yamada, L. Changes in seasonal evapotranspiration, soil water content, and crop coefficients in sugarcane, cassava, and maize fields in Northeast Thailand. Agricultural Water Management, v.67, p.133-143, 2004.

Wiedenfeld, B. Scheduling water application on drip irrigated sugarcane. Agricultural Water Management, v.64, p.169-181, 2004. 